OR "Angiomyofibroblastoma of the vulva with sarcomatous transformation".

Results A total of 69 reported cases were found. In our knowledge, the case presented is the first report of AMFB in Colombia. The age of the patients ranged between 16 and 82 years, with an average of 43 years. The main presenting symptom was the presence of a non-painful vulvar mass, although some of the patients in the reported cases reported pain and dyspareunia (8/69). The evolution time varied between 1 month and 9 years with an average of 21 months, and the size between 1 and 37 centimeters with an average of 7 centimeters. Of the cases taken to immunohistochemical study, $86 \%$ were positive for estrogen receptors and $80 \%$ for progesterone receptors, $88 \%$ were positive for desmin and $98 \%$ for vimentin, 92\% were negative for S100 and the cases that were positive corresponded to the lipomatous variant, $43 \%$ were positive for $\alpha$-SMA and 34\% for CD 34. Definitive diagnosis is based on histological findings. In this reviewed cases, only one relapse was reported. The universal treatment is excision and only one case of relapse was reported, which corresponded to the only reported case of malignant transformation.

Conclusion Vulvar AMFB is a rare mesenchymal tumor, with exclusive surgical treatment and excellent prognosis, although it may have malignant transformation. The reported case could represent the second case of AMFB with malignant transformation.

\section{ANGIOMYOFIBROBLASTOMA VULVAR: CASE REPORT AND LITERATURE REVIEW}

Sandra Marcela Buitrago, Carolina Morante Caicedo, Luis Orlando Puentes. Gynecology and Obstetric department. Pontifica Universidad Javeriana. Hospital Universitario San Ignacio, Bogotá (Colombia)

\subsection{6/ijgc-2020-ESGO.231}

Introduction/Background Angiomyofibroblastoma is part of the benign mesenchymal tumors of the genital tract and was first described by Fletcher in 1992. The vulva represents the main presentation site, but cases have been described at the level of the vagina, cervix, fallopian tubes, the scrotum and the ischiorectal fossa. It presents as a mass, generally painless, with well-defined edges and without compromising the overlying skin. It is normally less than $5 \mathrm{~cm}$, but cases of up to 37 $\mathrm{cm}$ have been described.

Methodology A 45-year-old woman with a 1 month history of painful vulvar mass of progressive growth. Physical examination reveals a stony, mobile mass of $5 \times 5 \mathrm{~cm}$ in right labia majora. Initial immunostaining suggested an AMFB, so she was taken to vulvectomy. A review was made of all articles in English or Spanish, published until March 10, 2019, related to the diagnosis and treatment of vulvar AMFB. The search included the PubMed, Embase, Cochrane, LILACS and Scielo databases, with the keywords "Vulvar Angiomyofibroblastoma" OR "Angiomyofibroblastoma of the vulva with sarcomatous transformation".

Results A total of 69 reported cases were found. In our knowledge, the case presented is the first report of AMFB in Colombia. The age of the patients ranged between 16 and 82 years, with an average of 43 years. The main presenting symptom was the presence of a non-painful vulvar mass, although some of the patients in the reported cases reported pain and dyspareunia (8/69). The evolution time varied between 1 month and 9 years with an average of 21 months, and the size between 1 and 37 centimeters with an average of 7 centimeters. Of the cases taken to immunohistochemical study, 86\% were positive for estrogen receptors and $80 \%$ for progesterone receptors, $88 \%$ were positive for desmin and 98\% for vimentin, 92\% were negative for S100 and the cases that were positive corresponded to the lipomatous variant, $43 \%$ were positive for $\alpha$-SMA and 34\% for CD 34. Definitive diagnosis is based on histological findings. In this reviewed cases, only one relapse was reported. The universal treatment is excision and only one case of relapse was reported, which corresponded to the only reported case of malignant transformation.

Conclusion Vulvar AMFB is a rare mesenchymal tumor, with exclusive surgical treatment and excellent prognosis, although it may have malignant transformation. The reported case could represent the second case of AMFB with malignant transformation.

Disclosures

\section{Organization of gynaecological cancer care}

\section{SIGNIFICANT VARIATION IN TREATMENT AND SURVIVAL OUTCOMES IN STAGE 2-4 OVARIAN CANCER IN ENGLAND: RESULTS FROM THE NATIONAL OVARIAN CANCER FEASIBILITY AUDIT PILOT}

${ }^{1}$ Sudha S Sundar, ${ }^{2}$ Craig Knott, ${ }^{2}$ Lizz Paley, ${ }^{3}$ Annwen Jones, ${ }^{4}$ Cary Wakefield, ${ }^{4}$ MarieClaire Platt, ${ }^{3}$ Rebecca Rennison, ${ }^{5}$ Jo Nieto, ${ }^{6}$ Andy Nordin. ${ }^{1}$ University of Birmingham; Institute of Cancer and Genomic Sciences; University of Birmingham; ${ }^{2}$ Ncras; ${ }^{3}$ Target Ovarian Cancer; ${ }^{4}$ Ovarian Cancer Action; ${ }^{5}$ Norwich Cancer Centre; ${ }^{6}$ Kent Cancer Centre

\subsection{6/ijgc-2020-ESG0.232}

Introduction/Background Complete cytoreductive surgery and platinum-based chemotherapy is standard of care in the United Kingdom (NICE, 2011), yet studies indicate substantial variation in the utilization of both (Kumar et al, 2016, Hall et al). Recent work from the Netherlands shows variations in treatment for ovarian cancer across regions; however, contribution to survival was unclear (Timmermans et al, 2019). Care that is not compliant with guidelines is also seen in other countries, including the USA (Warren et al, 2017).

As part of the Ovarian Cancer Audit Feasibility Pilot, geographic variation in treatment was investigated with the objective of informing improvements in treatment and outcomes for all women diagnosed with ovarian cancer in England.

Methodology Ovary, fallopian tube and primary peritoneal carcinomas ('ovarian cancers') diagnosed between January 2016 and December 2018 were audited using data extracted from the national cancer registry (Henson et al,). Borderline tumours were excluded. Data is routinely collected for every patient with cancer in England through a national dataset; Cancer outcomes and Services Dataset (COSD). This information was supplemented with relevant data from the Systemic Anti-Cancer Therapy (SACT) dataset for patients receiving chemotherapy and Hospital Episode Statistics (HES) for admitted patients. Linear probability models were constructed adjusting for tumour morphology, stage at diagnosis, patient age at diagnosis; Charlson comorbidity index, area income 


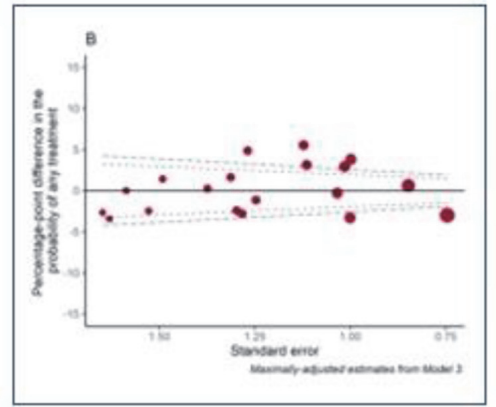

1A. Treatment Variation by Cancer Alliance:

each Alliance is reported relative to the average odds of any treatment within England:

cohort average $=73.8 \%$

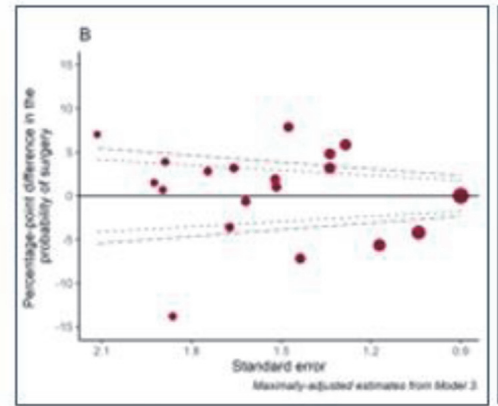

1B. Treatment Variation by Cancer Alliance:

each Alliance is reported relative to the average odds of any surgery within England:

cohort average $=51.0 \%$

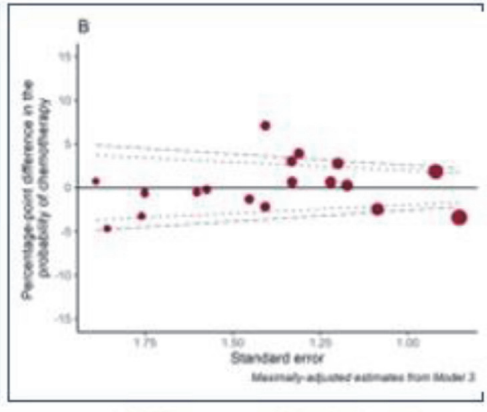

1C. Treatment Variation by Cancer Alliance:

each Alliance is reported relative to the average odds of any chemotherapy within England: cohort average $=66.5 \%$

Abstract 604 Figure 1 Variation in treatment for women with advanced OC

Data from 13,889 women diagnosed between 2016-2018, figures from Treatment variation report of the National Ovarian Cancer Feasibility pilot audit

Fig 1A. Variation in receiving any anticancer treatment

Fig 18. Variation in receiving surgery

Fig 1C. Variation in receiving chemotherapy

Outer Dashed lines indicate 3 standard deviations

\begin{tabular}{|c|c|c|c|c|c|c|}
\hline \multirow[b]{2}{*}{ Variabies } & \multicolumn{2}{|c|}{$\begin{array}{l}\text { Model } 1^{*} \\
\text { (estsaag) }\end{array}$} & \multicolumn{2}{|c|}{ 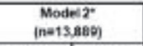 } & \multicolumn{2}{|c|}{$\begin{array}{l}\text { Model } 3^{*} \\
\text { \{n=13,85y }\end{array}$} \\
\hline & Esomate & Pralue & Esameno & p-value & Estimate & p-value \\
\hline Cohort average lintercepg) & 51.0 & 0.000 & 51.0 & 0.000 & 51.0 & 0.000 \\
\hline Cheshite and Mersersich & 0.7 & 0.73 & 0.6 & $0 \mathrm{es3}$ & 10 & 0.495 \\
\hline Eost Midands & -7.7 & 0.000 & 5.6 & 0.000 & 5.6 & 0.000 \\
\hline East of Enctand & 1,4 & 0.225 & 0.5 & 0.568 & 00 & 0.993 \\
\hline Greater Manchesser & 0.2 & 0.006 & -4.7 & 0.004 & 30 & 0.031 \\
\hline Humber, Coost and Vale & 5.2 & 0.030 & 3.8 & 0.044 & 39 & 0.038 \\
\hline Kentand We oway & 0.9 & 0.701 & 1.2 & 0.503 & 0.7 & 0.714 \\
\hline Lancastireand south Cumbria & 2.8 & 0.243 & 1.0 & 0.613 & 1.5 & 0.434 \\
\hline North Centrat and North Eass London & 0.8 & 0.000 & 1.1 & 0.544 & 2.9 & $0.10 \mathrm{e}$ \\
\hline North East and Cumbris & 4.8 & 0.000 & 2.3 & 0.096 & 32 & 0.017 \\
\hline North West and Scuen West London & 10.7 & 0.000 & 74 & 0.000 & 79 & 0.000 \\
\hline Peninaula & 5.8 & 0.004 & 2.3 & 0.134 & 1.9 & 0.211 \\
\hline Somerse Wilkshire, Avon, Gloucestershire & 6.0 & 0.000 & 5.9 & 0.000 & 48 & 0000 \\
\hline South East London & 13.5 & 0.000 & 9.8 & 0.007 & 70 & 0.001 \\
\hline Soutth Yorkshire, Bossettaw, Korth Derbyghire end Hardwick & -16.1 & 0.000 & -143 & 0.000 & $-1 \pm 8$ & 0.000 \\
\hline Surrey and Eussex & 4.2 & 0.000 & 7.1 & 0.000 & 5.9 & 0.000 \\
\hline Themes Valley & 4.3 & 0.030 & 4.5 & 0.007 & 32 & 0.054 \\
\hline Wessex $x$ & 12.2 & 0.000 & 6.2 & 0.000 & .72 & 0.000 \\
\hline West Midlands & 6.2 & 0.000 & 4.8 & 0.000 & 42 & 0.000 \\
\hline West Yorkskire and Harrogonte & 0.1 & 0.676 & -0.9 & 0.562 & -0.6 & 0.694 \\
\hline
\end{tabular}

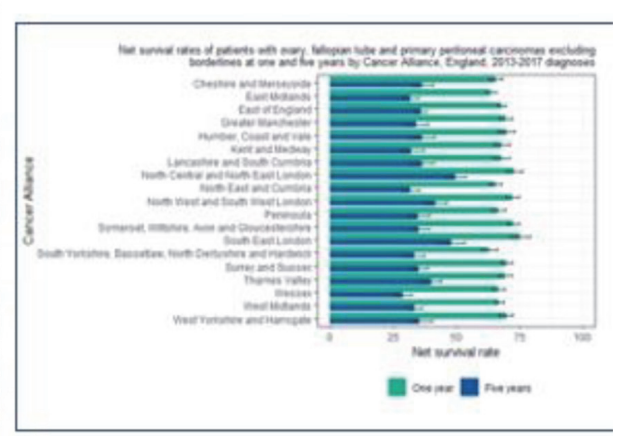

Model -1, unadjusted data, Model -2 adjusted for age, stage, tumour morphology, Model -3 as per Model 2 plus adjusting for comorbidity and deprivation. Areas in red are 3 standard deviation below average, areas in red are 3 standard deviation above average.

Abstract 604 Figure 2 A) Variation across 19 Cancer Alliances in England for patients with advanced OC receiving surgery: women diagnosed 2016-2018

B) Survival analysis from 19 Cancer alliances in England women diagnosed 2013-2017

Interpretation - Stressing differences in the time coverage \& cohort definitions, cross-referencing treatment variation \& survival analyses suggests Cancer Alliances less likely to undertake surgery had generally lower than average five year survival

deprivation. Tumours with stage 1 disease at diagnosis were excluded from analysis of variation in treatment. Treatment variations across the 19 cancer alliances (units of geography) were evaluated. Survival analyses were extracted from a previous cohort diagnosed 2013-2017.

Results Treatment received in 13,889 ovarian cancers was analysed. The weighted average probability (range for cancer alliances) of a stage 2-4 ovarian cancer receiving any treatment, any surgery, and any chemotherapy across England was $73.8 \%$ $(70.4 \%-79.3 \%), 51 \%(37.2 \%-58.9 \%)$ and $66.5 \%(61.8 \%-$ $73.6 \%$ ) respectively (figure 1 ). One-year net survival for the 19 Cancer Alliances in England varied between 62.9\% and
$75.2 \%$, 5-year net survival varied between $28.6 \%$ and $49.6 \%$. Cancer Alliances that were statistically less likely to undertake surgery generally had lower than average survival (figure 2).

Conclusion Significant variation in treatment and survival across England are demonstrated in this audit. The population-based nature of this robust audit indicates that our findings are likely to be relevant to international settings. Efforts to understand and reduce variation in treatment decision making and reducing the proportion of women not receiving treatment are critical to improving survival in ovarian cancer. Ongoing audit of treatment will be key to driving and monitoring progress. 